\title{
Virtual screen for repurposing approved and experimental drugs for candidate inhibitors of EBOLA virus infection
}

\section{[version 1; peer review: 2 approved]}

\author{
Veljko Veljkovic1, Philippe M. Loiseau (iD)2, Bruno Figadere (D2, Sanja Glisic², \\ Nevena Veljkovic'1, Vladimir R. Perovic', David P. Cavanaugh (D)3, \\ Donald R. Branch4
}

\footnotetext{
${ }^{1}$ Center for Multidisciplinary Research, University of Belgrade, Institute of Nuclear Sciences VINCA, P.O. Box 522, 11001 Belgrade, Serbia

${ }^{2}$ Antiparasitic Chemotherapy, UMR 8076 CNRS BioCIS, Faculty of Pharmacy Université Paris-Sud, Rue Jean-Baptiste Clément, F 92290- Chatenay-Malabry, France

${ }^{3}$ Bench Electronics, Bradford Dr., Huntsville, AL, 35801, USA

${ }^{4}$ Canadian Blood Services, Center for Innovation, 67 College Street, Toronto, Ontario, M5G 2M1, Canada
}

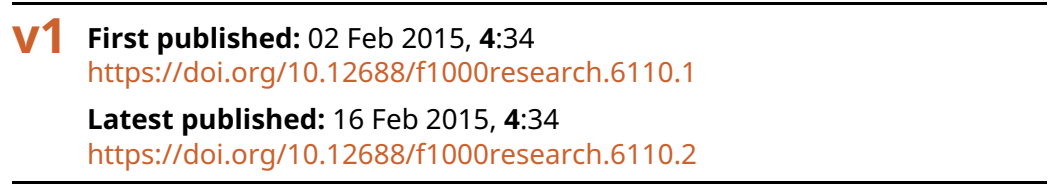

\section{Abstract}

The ongoing Ebola virus epidemic has presented numerous challenges with respect to control and treatment because there are no approved drugs or vaccines for the Ebola virus disease (EVD). Herein is proposed simple theoretical criterion for fast virtual screening of molecular libraries for candidate inhibitors of Ebola virus infection. We performed a repurposing screen of 6438 drugs from DrugBank using this criterion and selected 267 approved and 382 experimental drugs as candidates for treatment of EVD including 15 anti-malarial drugs and 32 antibiotics. An open source Web server allowing screening of molecular libraries for candidate drugs for treatment of EVD was also established.

\section{Keywords}

Ebola virus, drug candidates, entry inhibitors, virtual screening

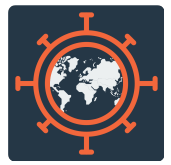

This article is included in the Emerging Diseases and Outbreaks gateway.

\section{Open Peer Review}

Approval Status

1 2

version 2

(revision)

16 Feb 2015

version 1

02 Feb 2015

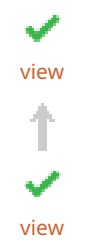

view

1. Patrick Butaye, Ross University, Basseterre,

Saint Kitts and Nevis

2. Bruno Botta, Sapienza University of Rome,

Rome, Italy

Any reports and responses or comments on the article can be found at the end of the article. 


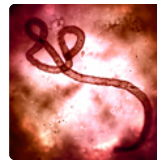

This article is included in the Ebola Virus

collection.

Corresponding author: Veljko Veljkovic (vv@vinca.rs)

Competing interests: No competing interests were disclosed.

Grant information: This work was supported by the Ministry of Education, Science and Technological Development of the Republic of Serbia (Grant no. 173001).

Copyright: ( 2015 Veljkovic V et al. This is an open access article distributed under the terms of the Creative Commons Attribution License, which permits unrestricted use, distribution, and reproduction in any medium, provided the original work is properly cited. Data associated with the article are available under the terms of the Creative Commons Zero "No rights reserved" data waiver (CC0 1.0 Public domain dedication).

How to cite this article: Veljkovic V, Loiseau PM, Figadere B et al. Virtual screen for repurposing approved and experimental drugs for candidate inhibitors of EBOLA virus infection [version 1; peer review: 2 approved] F1000Research 2015, 4:34 https://doi.org/10.12688/f1000research.6110.1

First published: 02 Feb 2015, 4:34 https://doi.org/10.12688/f1000research.6110.1 


\section{Introduction}

The current Ebola virus outbreak is one of the largest outbreaks of its kind in history and the first in West Africa. By January 14, 2015, a total of 21296 probable and confirmed cases, including 8429 deaths from Ebola virus disease (EVD), had been reported from five countries in West Africa - Guinea, Liberia, Nigeria, Senegal, and Sierra Leone (http://apps.who.int/iris/bitstream/10665/ 148237/2/roadmapsitrep_14Jan2015_eng.pdf?ua=1). EVD with a high case-fatality rate of $40 \%$ and with currently no approved vaccine or therapy, represents a major public health threat.

In response to the current Ebola virus outbreak, the international community has urged for accelerated development of drugs against EVD but also has endorsed the clinical use of unregistered treatments for Ebola ${ }^{1}$. Conventional time and the money consuming approach of drug development ( $>10$ years; $>2$ billions $\$$ ) does not meet the current urgent need for anti-Ebola drugs. Repurposing or repositioning of existing drugs could overcome some of these obstacles and help in the rapid discovery and development of therapeutics for EVD, although this approach does not negate the need for some preclinical studies and clinical trials for validation of the proposed indications. Recently, results of two large repurposing screenings of Food and Drug Administration (FDA)approved drugs have been reported. In the first study, Madrid and co-workers performed in vitro and in vivo (in mice) screening of 1012 FDA-approved drugs and selected 24 candidate entry inhibitors for Ebola virus ${ }^{2}$. In the second study, 53 inhibitors of Ebola virus infection with $\mathrm{IC}_{50}<10 \mu \mathrm{M}$ and selectivity index SI $>10$-fold have been identified by in vitro screening of 2816 FDA-approved drugs $^{3}$. In the same study, an additional 95 drugs which are active against Ebola virus infection with $\mathrm{IC}_{50}>10 \mu \mathrm{M}$ and SI $<10$-fold were also reported.

Although in vitro and in vivo screening for repurposing/repositioning of existing drugs could significantly accelerate discovery of new drugs these approaches are time-consuming and costly for screening of large drug libraries. Recently, we proposed a novel approach for in silico screening of molecular libraries for drug candidates $^{4-8}$. This approach, which uses the average quasi valence number (AQVN) and the electron-ion interaction potential (EIIP), parameters determining long-range interaction between biological molecules, might hold a key to overcoming some of these obstacles in experimental screening by significantly reducing the number of compounds which should be in vitro and in vivo tested ${ }^{9}$.

Herein, 267 approved and 382 experimental drugs, selected by the EIIP/AQVN-based virtual screening of DrugBank (http://www. drugbank.ca), have been proposed as candidate drugs for treatment of EVD. An open access portal allowing screening of molecular libraries for candidate drugs for treatment of EVD was established.

\section{Material and methods}

Molecular libraries

For screening of drugs for repurposing to select candidates for Ebola virus entry inhibitors, 1463 approved and 4975 experimental drugs from DrugBank (http://www.drugbank.ca) were screened. For development of the predictive criterion used in this analysis, the learning set (Dataset 1) encompassing 152 drugs which are selected as inhibitors of Ebola virus infection by in vitro and in vivo screening of 3828 FDA-approved drugs ${ }^{2,3}$, was established. As control data sets 45,010,644 compounds from PubChem (http://www.ncbi.nlm. nih.gov/pccompound) and 49 Ebola virus entry inhibitors collected by data mining of literature and patents, were used. For screening of literature data the NCBI literature database PubMed (http://www. ncbi.nlm.nih.gov/pubmed) was used. For search of patents and patent applications we used the Free Patent Online browser (http:// www.freepatentsonline.com).

Drug repurposing screen to identify active compounds that block Ebola entry

Specific recognition and targeting between interacting biological molecules at distances $>5 \AA$ are determined by the average AQVN and the EIIP ${ }^{10}$, which are derived from the general model pseudopotential ${ }^{11,12}$. These parameters for organic molecules are determined by the following simple equations ${ }^{10}$ :

$$
E I I P=0.25 \frac{Z^{*} \sin \left(1.04 \pi Z^{*}\right)}{2 \pi}
$$

Where $\mathrm{Z}^{*}$ is the average quasi-valence number (AQVN) determined by

$$
Z^{*}=\frac{1}{N} \sum_{i=1}^{m} n_{i} Z_{i}
$$

where $\mathrm{Z}_{i}$ is the valence number of the $i$-th atomic component, $\mathrm{n}_{i}$ is the number of atoms of the $i$-th component, $m$ is the number of atomic components in the molecule, and $\mathrm{N}$ is the total number of atoms. EIIP values calculated according to equation 1 and equation 2 are expressed in Rydberg units (Ry).

Among 3300 currently used molecular descriptors, AQVN and EIIP represent the unique physical properties which characterize the long-range interactions between biological molecules ${ }^{10}$. Small molecules with similar AQVN and EIIP values interact with the common therapeutic target, which allow establish criterions for virtual screening of molecular libraries for compounds with similar therapeutic properties ${ }^{4-9}$. Here we develop the EIIP/AQVN-based criterion for virtual screening of molecular libraries for candidate drugs against Ebola virus infection.

\section{Results and discussion}

Previously, analyses of the EIIP/AQVN distribution of 45,010,644 compounds from the PubChem database (http://www.ncbi.nlm. nih.gov/pccompound) revealed that $92.5 \%$ of presented compounds are homogenously distributed within EIIP and AQVN intervals $(0.00-0.11 \mathrm{Ry})$ and $(2.4-3.3)$, respectively). This domain of the EIIP/AQVN space, encompassing the majority of known 
chemical compounds, is referred to as the "basic EIIP/AQVN chemical space" $(\mathrm{BCS})^{6}$. Analysis of the molecular training set (Dataset 1), encompassing 152 small molecule inhibitors of Ebola virus infection selected by in vitro screening of 3828 FDA approved drugs ${ }^{2,3}$, show that $79 \%$ of these compounds are placed within AQVN and EIIP region $(2.3-2.7)$ and (0.0829-0.0954 Ry), respectively ("Ebola Virus Infection Inhibitors Space", EVIIS). The AQVN region $(2.36-2.54)$ and the EIIP region $(0.0912-0.0924$ Ry) form the part of EVIIS which encompasses $55.5 \%$ of all drugs from the learning set (core EVIIS, cEVIS). Literature data mining reveals 49 compounds with experimentally proved activity against Ebola virus infection (Table 1) ${ }^{13-29}$. Most of these compounds 47 (95.9\%) are placed within EVIIS (Table 1). Of note is that EVIIS and cEVIIS domains contain only $14.6 \%$ and $6.5 \%$ of compounds from PubChem, respectively. This confirms high specificity of clustering of Ebola virus infection inhibitors within the EIIP/AQVN space. Comparison of distributions of Ebola virus infection inhibitors and compounds from PubMed is given in Figure 1.

\section{Dataset 1. FDA-approved drugs which are active against Ebola virus infection ${ }^{2,3}$ \\ http://dx.doi.org/10.5256/f1000research.6110.d42876 \\ AQVN: average quasivalence number; EIIP: electron-ion interaction potential}

It was shown that Ebola virus glycoprotein (GP)-mediated entry and infection is subordinated with a membrane-trafficking event that translocates a GP binding partner to the cell surface, which depends on microtubules ${ }^{30,31}$. Consistently, microtubule inhibitors which block this trafficking process could decrease infection without interfering with the direct binding and translocation of the Ebola virus into cells. AQVN and EIIP values of microtubule modulators and transcription inhibitors with reported anti-Ebola virus activity are given in Table 2. As can be seen, all these compounds, which do not directly affect binding and internalization of Ebola virus, are located outside of EVIIS. This additionally confirms the specificity of the EVIS domain.

In further analysis we used EVIIS as a filter for virtual screening for candidate Ebola virus infection inhibitors. In Dataset 2622 approved and 1089 experimental drugs in Dataset 3 selected by EVIIS screening of 6532 drugs from DrugBank are reported. Using cEVIIS, we located 267 approved and 382 experimental drugs. This small molecular library represents a source of candidate drugs for treatment of Ebola virus disease (EVD), which can be further experimentally tested.

\section{Dataset 2. Approved and experimental drugs selected as candidate for treatment of EVD}

http://dx.doi.org/10.5256/f1000research.6110.d42877

AQVN: average quasivalence number; EIIP: electron-ion interaction potential
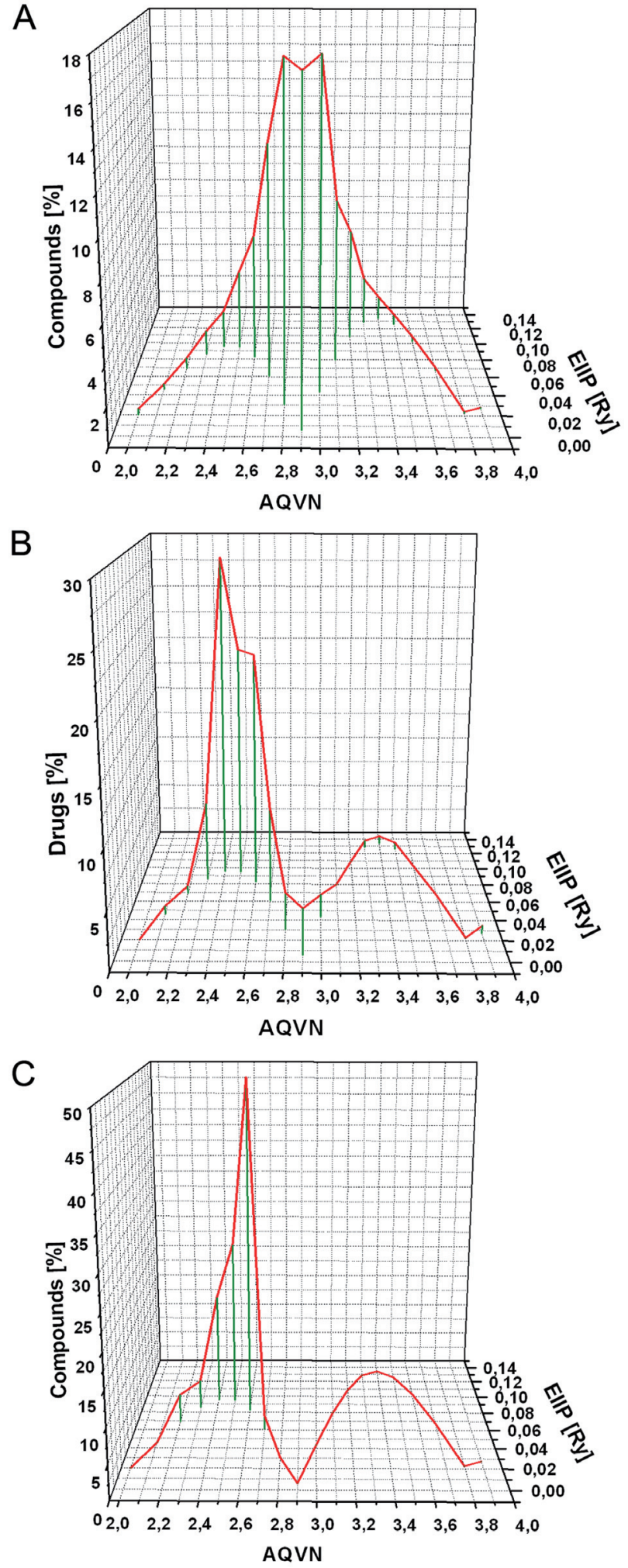

Figure 1. Distribution of compounds according to their average quasivalence number (AQVN) and electron-ion interaction potential (EIIP) values. (A) 45010644 compounds from the PubChem database (http://www.ncbi.nlm. nih.gov/pccompound); (B) FDA-approved drugs which are active against Ebola virus infection (Dataset 1) $)^{2,3}$; (C) Entry inhibitors of Ebola virus (Table 1). 
Table 1. Small-molecule entry inhibitors for Ebola virus.

\begin{tabular}{|c|c|c|c|c|}
\hline Compound & Formula & AQVN & EIIP [Ry] & Reference \\
\hline Chloroquine & C18H26CIN3 & 2.375 & 0.0941 & 16 \\
\hline Bafilomycin A1 & $\mathrm{C} 35 \mathrm{H} 58 \mathrm{O} 9$ & 2.471 & 0.0960 & 17 \\
\hline Cytochalasin B & C29H37NO5 & 2.611 & 0.0810 & 17 \\
\hline Cytochalasin D & С30H37NO6 & 2.676 & 0.0672 & 17 \\
\hline Latruculion A & C22H31NO5S & 2.667 & 0.0693 & 17 \\
\hline Jasplakinolide & $\mathrm{C} 36 \mathrm{H} 45 \mathrm{BrN} 4 \mathrm{O} 6$ & 2.674 & 0.0676 & 18 \\
\hline Clomiphene & C26H28CINO & 2.526 & 0.0926 & 18 \\
\hline Toremifene & $\mathrm{C} 26 \mathrm{H} 28 \mathrm{CINO}$ & 2.526 & 0.0926 & 18 \\
\hline Chlorpromazine & C17H19CIN2S & 2.600 & 0.0829 & 19 \\
\hline Amiodarone & $\mathrm{C} 25 \mathrm{H} 2912 \mathrm{NO} 3$ & 2.567 & 0.0880 & 20 \\
\hline Dronedarone & $\mathrm{C} 31 \mathrm{H} 44 \mathrm{~N} 2 \mathrm{O} 5 \mathrm{~S}$ & 2.578 & 0.0864 & 20 \\
\hline Verapamil & $\mathrm{C} 27 \mathrm{H} 38 \mathrm{~N} 2 \mathrm{O} 4$ & 2.535 & 0.0917 & 20 \\
\hline Clomiphene & $\mathrm{C} 26 \mathrm{H} 28 \mathrm{CINO}$ & 2.526 & 0.0926 & 21 \\
\hline AY-9944 & $\mathrm{C} 22 \mathrm{H} 28 \mathrm{Cl} 2 \mathrm{~N} 2$ & 2.370 & 0.0938 & 21 \\
\hline Ro 48-8071 & $\mathrm{C} 23 \mathrm{H} 27 \mathrm{BrFNO} 2$ & 2.505 & 0.0940 & 21 \\
\hline U18666A & $\mathrm{C} 25 \mathrm{H} 41 \mathrm{NO} 2$ & 2.290 & 0.0849 & 21 \\
\hline Terconazole & $\mathrm{C} 26 \mathrm{H} 31 \mathrm{Cl} 2 \mathrm{~N} 5 \mathrm{O} 3$ & 2.687 & 0.0644 & 21 \\
\hline Triparanol & $\mathrm{C} 27 \mathrm{H} 32 \mathrm{CINO} 2$ & 2.508 & 0.0941 & 21 \\
\hline Impramine & $\mathrm{C} 19 \mathrm{H} 24 \mathrm{~N} 2$ & 2.444 & 0.0964 & 22 \\
\hline 3.47 & $\mathrm{C} 34 \mathrm{H} 43 \mathrm{~N} 3 \mathrm{O} 5$ & 2.635 & 0.0763 & 23 \\
\hline Cytochalasin B & C29H37NO5 & 2.611 & 0.0810 & 24 \\
\hline Cytochalasin D & С30H37NO6 & 2.676 & 0.0672 & 24 \\
\hline Latrunculin A & C22H31NO5S & 2.667 & 0.0693 & 24 \\
\hline Jasplakinolide & $\mathrm{C} 36 \mathrm{H} 45 \mathrm{BrN} 4 \mathrm{O} 6$ & 2.674 & 0.0676 & 24 \\
\hline NSC62914 & $\mathrm{C} 31 \mathrm{H} 40 \mathrm{O} 3$ & 2.460 & 0.0962 & 25 \\
\hline Compound 1 & $\mathrm{C} 30 \mathrm{H} 38 \mathrm{~N} 6 \mathrm{O} 2$ & 2.632 & 0.0770 & 26 \\
\hline Compound 2 & $\mathrm{C} 32 \mathrm{H} 46 \mathrm{~N} 6$ & 2.429 & 0.0963 & 26 \\
\hline Compound 3 & $\mathrm{C} 28 \mathrm{H} 34 \mathrm{~N} 6 \mathrm{O} 2$ & 2.686 & 0.0635 & 26 \\
\hline Compound 5 & $\mathrm{C} 42 \mathrm{H} 58 \mathrm{~N} 10 \mathrm{O} 6$ & 2.690 & 0.0635 & 27 \\
\hline Compound $8 a$ & $\mathrm{C} 17 \mathrm{H} 23 \mathrm{~N} 3 \mathrm{O} 3$ & 2.695 & 0.0621 & 27 \\
\hline Compound 8b & $\mathrm{C} 17 \mathrm{H} 23 \mathrm{~N} 3 \mathrm{O} 3$ & 2.695 & 0.0621 & 27 \\
\hline Compound 8y & $\mathrm{C} 16 \mathrm{H} 20 \mathrm{BrNO} 2$ & 2.610 & 0.0812 & 27 \\
\hline Compound $15 \mathrm{~h}$ & C15H20JN5O & 2.667 & 0.0693 & 27 \\
\hline Compound 15k & $\mathrm{C} 15 \mathrm{H} 128 \mathrm{Br} 3 \mathrm{~N} 5 \mathrm{O}$ & 2.667 & 0.0693 & 27 \\
\hline Retinazone & C38H56Na3N5S2 & 2.385 & 0.0947 & 28 \\
\hline Compound 7 & $\mathrm{C} 17 \mathrm{H} 12 \mathrm{~F} 4 \mathrm{~N} 2$ & 2.467 & 0.0647 & 29 \\
\hline Brincidofovir* & C27H52N3O7P & 2.467 & 0.0961 & 30 \\
\hline Hit compound 3 & $\mathrm{C} 25 \mathrm{H} 35 \mathrm{~N} 3 \mathrm{O} 2$ & 2.494 & 0.0950 & 31 \\
\hline Hit compound 3.1 & $\mathrm{C} 21 \mathrm{H} 24 \mathrm{CIN} 3 \mathrm{O} 2$ & 2.667 & 0.0693 & 31 \\
\hline Hit compound 3.2 & $\mathrm{C} 2 \mathrm{OH} 29 \mathrm{~N} 3 \mathrm{O} 2$ & 2.518 & 0.0933 & 31 \\
\hline Hit compound 3.3 & $\mathrm{C} 30 \mathrm{H} 35 \mathrm{~N} 3 \mathrm{O} 2$ & 2.556 & 0.0894 & 31 \\
\hline Hit compound 3.4 & $\mathrm{C} 25 \mathrm{H} 32 \mathrm{~N} 4 \mathrm{O} 3$ & 2.656 & 0.0717 & 31 \\
\hline Hit compound 3.5 & $\mathrm{C} 2 \mathrm{OH} 23 \mathrm{~N} 3 \mathrm{O} 2$ & 2.708 & 0.0587 & 31 \\
\hline Hit compound 3.6 & $\mathrm{C} 25 \mathrm{H} 33 \mathrm{~N} 3 \mathrm{O} 2$ & 2.540 & 0.09913 & 31 \\
\hline Hit compound 3.7 & $\mathrm{C} 22 \mathrm{H} 27 \mathrm{~N} 3 \mathrm{O} 3$ & 2.691 & 0.0633 & 31 \\
\hline Hit compound 3.18 & $\mathrm{C} 26 \mathrm{H} 37 \mathrm{~N} 3 \mathrm{O} 2$ & 2.471 & 0.0471 & 31 \\
\hline Hit compound 3.48 & $\mathrm{C} 34 \mathrm{H} 43 \mathrm{~N} 3 \mathrm{O} 5$ & 2.635 & 0.0763 & 31 \\
\hline Hit compound 3.105 & $\mathrm{C} 34 \mathrm{H} 40 \mathrm{~N} 6 \mathrm{O} 2$ & 2.658 & 0.0712 & 31 \\
\hline NSC 62914 & $\mathrm{C} 31 \mathrm{H} 3903$ & 2.480 & 0.0957 & 32 \\
\hline
\end{tabular}

*Experimental drug applied for treatment of Ebola patients in Liberia (http://www.ox.ac.uk/ news/2014-11-13-oxford-lead-trial-experimental-drug-ebola-patients) 
Table 2. Viral transcription inhibitors and microtubule modulators with anti-Ebola virus activity.

\begin{tabular}{|c|c|c|c|}
\hline Compound & Formula & AQVN & EIIP $[R y]$ \\
\hline \multicolumn{4}{|c|}{ Viral transcription inhibitors } \\
\hline $\mathrm{BC} \times 4430$ & C11H15N5O3 & 3.000 & 0.0439 \\
\hline Favipiravir & C5H4FN3O2 & 3.467 & 0.1304 \\
\hline C-c3Ado & $\mathrm{C} 12 \mathrm{H} 16 \mathrm{~N} 4 \mathrm{O} 3$ & 2.914 & 0.0112 \\
\hline c3Nep & $\mathrm{C} 12 \mathrm{H} 14 \mathrm{~N} 4 \mathrm{O} 3$ & 3.030 & 0.0552 \\
\hline "D-like" 1'-6'-isoneplanocin & $\mathrm{C} 11 \mathrm{H} 12 \mathrm{~N} 5 \mathrm{O} 3$ & 3.194 & 0.1076 \\
\hline "L-like" 1'-6'-isoneplanocin & C11H12N5O3 & 3.194 & 0.1076 \\
\hline CMLDBU3402 & C30H26BrN3O7 & 3.045 & 0.1343 \\
\hline \multicolumn{4}{|c|}{ Microtubule modulators } \\
\hline Vinblastine & $\mathrm{C} 13 \mathrm{H} 8 \mathrm{Cl} 2 \mathrm{~N} 2 \mathrm{O} 4$ & 3.310 & 0.0130 \\
\hline Vinorelbine & $\mathrm{C} 45 \mathrm{H} 54 \mathrm{~N} 4 \mathrm{O} 8$ & 2.721 & 0.0552 \\
\hline Vincristine & $\mathrm{C} 46 \mathrm{H} 56 \mathrm{~N} 4 \mathrm{O} 10$ & 2.759 & 0.0439 \\
\hline Colchicine & C22H25NO6 & 2.852 & 0.0121 \\
\hline Nocodazole & $\mathrm{C} 14 \mathrm{H} 11 \mathrm{~N} 3 \mathrm{O} 3 \mathrm{~S}$ & 3.312 & 0.1298 \\
\hline Mebendazole & $\mathrm{C} 16 \mathrm{H} 13 \mathrm{~N} 3 \mathrm{O} 3$ & 3.143 & 0.0934 \\
\hline Albendazole & C12H15N3O2S & 2.909 & 0.0092 \\
\hline
\end{tabular}

Dataset 3. Experimental drugs selected as candidate for treatment of EVD

http://dx.doi.org/10.5256/f1000research.6110.d42878

AQVN: average quasivalence number; EIIP: electron-ion interaction potential

Madrid and co-workers selected 24 drugs by in vitro screening of 1012 FDA-approved drugs, which are effective against Ebola virus infection ${ }^{2}$. They also showed that among these compounds, four antimalarial drugs (chloroquine, hydroxychloroquine, amodiaquine and aminoquinoline-13) also are effective against Ebola virus infection in vivo ${ }^{2}$. Among 53 compounds which effectively inhibit Ebola virus infection in vitro, which Kouznetsova and co-workers selected from 2816 approved drugs, are also three antimalarial drugs (mefloquione, chloroquine, amodiaquine) ${ }^{3}$. It was also suggested that application of chloroquine for prevention of virus transmission should be considered because this compound significantly inhibits Ebola virus infection ${ }^{13}$. Our analysis showed that 15 of 22 approved ant-malarial drugs (http://en.wikipedia. org/wiki/Antimalarial_medication) are located in EVIIS (Table 3). Six 2-alkylquinolines have been also included in this study. This chemical series is promising as some derivatives exhibited antiviral activity such as $2 \mathrm{PQ}$, and $2 \mathrm{QQ}^{32,33}$ antimalarial activity such as $2 \mathrm{PQ}$ and $2 \mathrm{PentQ}^{34}$, antileishmanial activity such as $2 \mathrm{PQ}^{35,36}$ and neurotrophin-like activity on dopaminergic neurons such as $2 \mathrm{QI} 15^{37}$. These compounds exhibit some advantages in regard to their chemical synthesis with few steps and good yields as well as their chemical stability in tropical conditions of storage. Their combined effects against virus and Leishmania parasites suggested they could be an advantage for the treatment of Leishmania/HIV co-infections and they were considered as attractive enough to enter the pipeline of DNDi on 2010.

All these data strongly suggest that this class of drugs should be further investigated as a promising source of therapeutics for treatment of EVD. Anti-malarial drugs with dual activity should be of special interest because malaria represents the highest health-related disease in African countries with EVD.

Among 3828 FDA-approved drugs screened for anti-Ebola activity were six antibiotics which inhibit Ebola virus infection (azthromycin, erythromycin, spiramycin, dirithromycin, maduramicin, charitromycin $)^{2,3}$. All these antibiotics are within EVIIS and four of them are in cEVIIS. Analysis of 184 approved antibiotics (Dataset 4) showed that only $32(17.4 \%)$ have AQVN and EIIP values in EVIIS, and that 11 of them are located within cEVIIS. Previously we reported domains of AQVN and EIIP which characterize different classes of antibiotics (Table 4$)^{6}$. According to these data, among antibiotics some macrolides, pleuromutilins and aminoglycosides have the highest chance for inhibition of Ebola virus infection. Of note is that five of six antibiotics with experimentally proved activity against Ebola virus infection (azthromycin, erythromycin, spiramycin, dirithromycin, charitromycin) are macrolides. Antibiotics representing candidate Ebola virus infection inhibitors selected by EIIP/AQVN criterion are given in Table 5. 
Dataset 4. Approved antibiotics screened for candidate anti-Ebola drugs

http://dx.doi.org/10.5256/f1000research.6110.d42879

AQVN: average quasivalence number; EIIP: electron-ion interaction potential

Previous, we determined AQVN and EIIP domains characterizing different classes of anti-HIV drugs ${ }^{4-9}$. As can be seen in Table 6, the EIIP/AQVN domain of CCR5 HIV entry inhibitors is within
EVIIS, and domains of CXCR4 HIV entry inhibitors and HIV protease inhibitors partially overlaps EVIIS. The EIIP/AQVN domains of other classes of anti-HIV agents are located outside EVIIS. This indicates that some HIV entry inhibitors and HIV protease inhibitors could also be effective drugs against Ebola virus infection.

In conclusion, the presented results show that the EIIP/AQVN criterion can be used as an efficient filter in virtual screening of molecular libraries for candidate inhibitors of Ebola virus infection. Approved (Dataset 2) and experimental drugs (Dataset 3), anti-malarial drugs (Table 3) and antibiotics (Table 5) selected by this criterion

Table 3. Approved anti-malarial drugs selected as candidate drugs for EVD.

\begin{tabular}{|l|l|l|l|}
\hline Compound & Formula & AQVN & EIIP [Ry] \\
\hline Quinine & C20H24N2O2 & 2.625 & 0.0784 \\
\hline Chloroquinine & C18H26CIN3 & 2.375 & 0.0941 \\
\hline Amodiquinine & C20H22CIN3O & 2.638 & 0.0756 \\
\hline Proguanil & $\mathrm{C} 11 \mathrm{H} 16 \mathrm{CIN5}$ & 2.606 & 0.0819 \\
\hline Mefloquine & $\mathrm{C} 17 \mathrm{H} 16 \mathrm{~F} 6 \mathrm{~N} 2 \mathrm{O}$ & 2.524 & 0.0928 \\
\hline Primaquine & $\mathrm{C} 15 \mathrm{H} 21 \mathrm{NO} 3$ & 2.600 & 0.0829 \\
\hline Halofantrine & $\mathrm{C} 26 \mathrm{H} 30 \mathrm{Cl} 2 \mathrm{~F} 3 \mathrm{NO}$ & 2.381 & 0.0945 \\
\hline Clindamycin & $\mathrm{C} 18 \mathrm{H} 33 \mathrm{CIN} 2 \mathrm{O} 5 \mathrm{~S}$ & 2.533 & 0.0919 \\
\hline Artemether & $\mathrm{C} 16 \mathrm{H} 26 \mathrm{O} 5$ & 2.553 & 0.0897 \\
\hline Piperaquine & $\mathrm{C} 29 \mathrm{H} 32 \mathrm{Cl} 2 \mathrm{~N} 6$ & 2.609 & 0.0814 \\
\hline Artemotil & $\mathrm{C} 17 \mathrm{H} 28 \mathrm{O} 5$ & 2.520 & 0.0931 \\
\hline Dihydroartemisin & $\mathrm{C} 15 \mathrm{H} 24 \mathrm{O} 5$ & 2.591 & 0.0844 \\
\hline Quinidine & $\mathrm{C} 20 \mathrm{H} 24 \mathrm{~N} 2 \mathrm{O} 2$ & 2.625 & 0.0784 \\
\hline Cinchonidine & $\mathrm{C} 19 \mathrm{H} 22 \mathrm{~N} 2 \mathrm{O}$ & 2.591 & 0.0844 \\
\hline Artemisin & $\mathrm{C} 15 \mathrm{H} 22 \mathrm{O} 5$ & 2.667 & 0.0693 \\
\hline
\end{tabular}

Table 4. AQVN and EIIP range of different antibiotics classes ${ }^{6}$.

\begin{tabular}{|l|l|l|}
\hline Antibiotic class & AQVN & EIIP [Ry] \\
\hline Penicillins & $2.975-3.180$ & $0.035-0.124$ \\
\hline Cephalosporins & $3.071-3.473$ & $0.070-0.130$ \\
\hline Carbapenems \& Penems & $2.973-3.059$ & $0.022-0.066$ \\
\hline Monobactams & $3.166-3.581$ & $0.100-0.134$ \\
\hline Quinolines & $2.760-3.060$ & $0.003-0.065$ \\
\hline Aminoglycosides & $2.552-2.820$ & $0.024-0.084$ \\
\hline Tetracyclines & $2.933-3.111$ & $0.018-0.084$ \\
\hline Macrolides & $2.467-2.630$ & $0.077-0.096$ \\
\hline Pleuromutilins & $2.395-2.473$ & $0.095-0.096$ \\
\hline Nitrofurans & $3.652-3.826$ & $0.010-0.086$ \\
\hline
\end{tabular}


Table 5. Antibiotics selected as candidate drugs for EVD.

\begin{tabular}{|c|c|c|c|}
\hline Antibiotics & Formula & $A Q V N$ & EIIP [Ry] \\
\hline Tiamulin & $\mathrm{C}_{28} \mathrm{H}_{47} \mathrm{NO}_{4} \mathrm{~S}$ & 2.395 & 0.095 \\
\hline Retapamulin & $\mathrm{C}_{30} \mathrm{H}_{47} \mathrm{NO}_{4} \mathrm{~S}$ & 2.434 & 0.096 \\
\hline Valnemulin & $\mathrm{C}_{31} \mathrm{H}_{52} \mathrm{~N}_{2} \mathrm{O}_{5} \mathrm{~S}$ & 2.440 & 0.096 \\
\hline Azithromycin & $\mathrm{C}_{38} \mathrm{H}_{72} \mathrm{~N}_{2} \mathrm{O}_{12}$ & 2.468 & 0.096 \\
\hline BC-3205 & $\mathrm{C}_{32} \mathrm{H}_{51} \mathrm{~N}_{2} \mathrm{O}_{5} \mathrm{~S}$ & 2.472 & 0.096 \\
\hline Dirithromycin & $\mathrm{C}_{42} \mathrm{H}_{78} \mathrm{~N}_{2} \mathrm{O}_{14}$ & 2.500 & 0.095 \\
\hline Clarithromycin & $\mathrm{C}_{38} \mathrm{H}_{69} \mathrm{NO}_{13}$ & 2.512 & 0.094 \\
\hline Surfactin & $\mathrm{C}_{53} \mathrm{H}_{93} \mathrm{~N}_{7} \mathrm{O}_{13}$ & 2.518 & 0.093 \\
\hline Erythromycin & $\mathrm{C}_{37} \mathrm{H}_{67} \mathrm{NO}_{13}$ & 2.525 & 0.093 \\
\hline Clindamycin & $\mathrm{C}_{18} \mathrm{H}_{33} \mathrm{ClN}_{2} \mathrm{O}_{5} \mathrm{~S}$ & 2.533 & 0.092 \\
\hline Roxithromycin & $\mathrm{C}_{41} \mathrm{H}_{76} \mathrm{~N}_{2} \mathrm{O}_{15}$ & 2.537 & 0.092 \\
\hline Oleandomycin & $\mathrm{C}_{35} \mathrm{H}_{61} \mathrm{NO}_{12}$ & 2.550 & 0.090 \\
\hline Gentamicin & $\mathrm{C}_{21} \mathrm{H}_{43} \mathrm{~N}_{5} \mathrm{O}_{7}$ & 2.553 & 0.090 \\
\hline Spiramycin & $\mathrm{C}_{43} \mathrm{H}_{74} \mathrm{~N}_{2} \mathrm{O}_{14}$ & 2.556 & 0.089 \\
\hline Mupirocin & $\mathrm{C}_{26} \mathrm{H}_{44} \mathrm{O}_{9}$ & 2.557 & 0.089 \\
\hline Lincomycin & $\mathrm{C}_{18} \mathrm{H}_{34} \mathrm{~N}_{2} \mathrm{O}_{6} \mathrm{~S}$ & 2.590 & 0.085 \\
\hline Netilmicin & $\mathrm{C}_{21} \mathrm{H}_{41} \mathrm{~N}_{5} \mathrm{O}_{7}$ & 2.595 & 0.084 \\
\hline Astromicin & $\mathrm{C}_{17} \mathrm{H}_{35} \mathrm{~N}_{5} \mathrm{O}_{6}$ & 2.603 & 0.082 \\
\hline Tylosin & $\mathrm{C}_{46} \mathrm{H}_{77} \mathrm{NO}_{17}$ & 2.610 & 0.081 \\
\hline Kitasamycin & $\mathrm{C}_{35} \mathrm{H}_{59} \mathrm{NO}_{13}$ & 2.611 & 0.081 \\
\hline Josamycin & $\mathrm{C}_{42} \mathrm{H}_{69} \mathrm{NO}_{15}$ & 2.614 & 0.080 \\
\hline Telithromycin & $\mathrm{C}_{43} \mathrm{H}_{65} \mathrm{~N}_{5} \mathrm{O}_{10}$ & 2.618 & 0.080 \\
\hline Telithromycin & $\mathrm{C}_{43} \mathrm{H}_{65} \mathrm{~N}_{5} \mathrm{O}_{10}$ & 2.618 & 0.080 \\
\hline Verdamicin & $\mathrm{C}_{20} \mathrm{H}_{39} \mathrm{~N}_{5} \mathrm{O}_{7}$ & 2.620 & 0.080 \\
\hline Midecamycin & $\mathrm{C}_{41} \mathrm{H}_{67} \mathrm{NO}_{15}$ & 2.629 & 0.078 \\
\hline Troleandomycin & $\mathrm{C}_{41} \mathrm{H}_{67} \mathrm{NO}_{15}$ & 2.629 & 0.078 \\
\hline Sisomicin & $\mathrm{C}_{19} \mathrm{H}_{37} \mathrm{~N}_{5} \mathrm{O}_{7}$ & 2.647 & 0.074 \\
\hline Cethromycin & $\mathrm{C}_{42} \mathrm{H}_{59} \mathrm{~N}_{3} \mathrm{O}_{10}$ & 2.649 & 0.073 \\
\hline Carbomycin A & $\mathrm{C}_{42} \mathrm{H}_{67} \mathrm{NO}_{16}$ & 2.667 & 0.069 \\
\hline Dibekacin & $\mathrm{C}_{18} \mathrm{H}_{37} \mathrm{~N}_{5} \mathrm{O}_{8}$ & 2.676 & 0.067 \\
\hline Echinocandin B & $\mathrm{C}_{52} \mathrm{H}_{81} \mathrm{~N}_{7} \mathrm{O}_{16}$ & 2.692 & 0.063 \\
\hline Rifabutin & $\mathrm{C}_{46} \mathrm{H}_{62} \mathrm{~N}_{4} \mathrm{O}_{11}$ & 2.699 & 0.061 \\
\hline
\end{tabular}

Table 6. AQVN and EIIP range of anti-HIV drugs ${ }^{6}$.

\begin{tabular}{|l|l|l|}
\hline Target & AQVN & EIIP [Ry] \\
\hline CXCR4 & $2.16-2.53$ & $0.062-0.096$ \\
\hline CCR5 & $2.42-2.63$ & $0.079-0.099$ \\
\hline PI & $2.61-2.78$ & $0.040-0.080$ \\
\hline NRTI/NtRTI & $2.92-3.20$ & $0.040-0.100$ \\
\hline INI & $3.00-3.20$ & $0.044-0.116$ \\
\hline Anti-HIV flavonoids & $3.34-3.59$ & $0.110-0.135$ \\
\hline
\end{tabular}

represents a valuable source of candidate therapeutics for treatment of EVD, some of which are already approved by FDA for treatment of other diseases which can be repurposed for use in EVD. We hope that these data, obtained by an in silico drug repurposing screen, will accelerate discovery of drugs for treatment of EVD, which are necessary in this ongoing emergency situation caused by the current unprecedented Ebola virus outbreak. To enable other researchers working on online EIIP/AQVN-based screening of different sources of small molecules for candidate Ebola drugs, we established an open web server (http://www.biomedconsulting. info/ebola_screen.php). 


\section{Data availability}

The virtual screen for candidate inhibitors of EBOLA virus infection web tool is available at: http://www.biomedconsulting.info/ tools/ebolascreen.php. An archived version can be accessed at: http://www.webcitation.org/6Vxtuojgx ${ }^{38}$

F1000Research: Dataset 1. FDA-approved drugs which are active against Ebola virus infection ${ }^{2,3}, 10.5256 /$ f1000research.6110.d4287639

F1000Research: Dataset 2. Approved and experimental drugs selected as candidate for treatment of EVD, 10.5256/f1000research.6110.d42877 40

F1000Research: Dataset 3. Experimental drugs selected as candidate for treatment of EVD, 10.5256/f1000research.6110.d42878

F1000Research: Dataset 4. Approved antibiotics screened for candidate anti-Ebola drugs, 10.5256/f1000research.6110.d42879 ${ }^{42}$
Author contributions

Conceived and designed the study: VV SG NV. Developed the analysis tools: VP. Analyzed the data: VV SG NV DRB PPML BF DPC. Wrote the paper: VV DRB PPML.

\section{Competing interests}

No competing interests were disclosed.

\section{Grant information}

This work was supported by the Ministry of Education, Science and Technological Development of the Republic of Serbia (Grant no. 173001).

\section{Acknowledgments}

The authors would like to gratefully acknowledge networking support by the COST Action CM1307.
1. Enserink M: Infectious diseases. Debate erupts on 'repurposed' drugs for Ebola. Science. 2014; 345(6198): 718-9. PubMed Abstract | Publisher Full Text

2. Madrid PB, Chopra S, Manger ID, et al.: A systematic screen of FDA-approved drugs for inhibitors of biological threat agents. PLoS One. 2013; 8(4): e60579. PubMed Abstract | Publisher Full Text | Free Full Text

3. Kouznetsova J, Sun W, Martínez-Romero C, et al.: Identification of $\mathbf{5 3}$ compounds that block Ebola virus-like particle entry via a repurposing screen of approved drugs. Emerg Microb Infect. 2014; 3: e84 Publisher Full Text

4. Veljkovic V, Veljkovic N, Este J, et al.: Application of the EIIP/ISM bioinformatics concept in development of new drugs. Curr Med Chem. 2007; 14(4): 441-53. PubMed Abstract | Publisher Full Text

5. Veljkovic V, Mouscadet JF, Veljkovic N, et al.: Simple criterion for selection of flavonoid compounds with anti-HIV activity. Bioorg Medic Chem Lett. 2007; 17(5): 1226-32.

PubMed Abstract | Publisher Full Text

6. Veljkovic N, Glisic S, Perovic V, et al.: The role of long-range intermolecular interactions in discovery of new drugs. Exp Opin Drug Disc. 2011; 6(12): 1263-70.

PubMed Abstract | Publisher Full Text

7. Maga G, Veljkovic N, Crespan E, et al:: New in silico and conventional in vitro approaches to advance HIV drug discovery and design. Exp Opin Drug Discov. 2013; 8(1): 83-92.

PubMed Abstract | Publisher Full Text

8. Veljkovic N, Glisic S, Prljic J, et al:: Simple and general criterion for "in silico" screening of candidate HIV drugs. Curr Pharm Biotechnol. 2013; 14(5): 561-9. PubMed Abstract | Publisher Full Text

9. Tintori C, Veljkovic N, Veljkovic V, et al.: Computational studies of the interaction between the HIV-1 integrase tetramer and the cofactor LEDGF/p75: insights from molecular dynamics simulations and the informational spectrum method. Proteins. 2010; 78(16): 3396-408.

PubMed Abstract | Publisher Full Text

10. Veljkovic V: A theoretical approach to preselection of carcinogens and chemical carcinogenesis. New York: Gordon \& Breach. 1980. Reference Source

11. Veljkovic V, Slavic I: Simple general-model pseudopotential. Phys Rev Lett. 1972; 29: 105-7. Publisher Full Text

12. Veljkovic V: The dependence of the Fermi energy on the atomic number. Phys Lett. 1973; 45A(1): 41-2. Publisher Full Text

13. Wool-Lewis RJ, Bates P: Characterization of Ebola virus entry by using pseudotyped viruses: identification of receptor-deficient cell lines. $J$ Virol. 1998; 72(4): 3155-60. PubMed Abstract | Free Full Text

14. Yonezawa A, Cavrois M, Greene WC: Studies of Ebola virus glycoproteinmediated entry and fusion by using pseudotyped human immunodeficiency virus type 1 virions: involvement of cytoskeletal proteins and enhancement by tumor necrosis factor alpha. $J$ Virol. 2005; 79(2): 918-26.

PubMed Abstract | Publisher Full Text | Free Full Text

15. Johansen LM, Brannan JM, Delos SE, et al:: FDA-approved selective estrogen receptor modulators inhibit Ebola virus infection. Sci Transl Med. 2013; 5(190): 190ra79.

PubMed Abstract | Publisher Full Text | Free Full Text

16. Bhattacharyya $S$, Warfield KL, Ruthel $G$, et al: Ebola virus uses clathrinmediated endocytosis as an entry pathway. Virology. 2010; 401(1): 18-28. PubMed Abstract | Publisher Full Text | Free Full Text

17. Gehring G, Rohrmann K, Atenchong N, et al.: The clinically approved drugs amiodarone, dronedarone and verapamil inhibit filovirus cell entry. J Antimicrob Chemother. 2014; 69(8): 2123-31.

PubMed Abstract | Publisher Full Text

18. Shoemaker CJ, Schornberg KL, Delos SE, et al.: Multiple cationic amphiphiles induce a Niemann-Pick $C$ phenotype and inhibit Ebola virus entry and infection. PloS One. 2013; 8(2): e56265.

PubMed Abstract | Publisher Full Text | Free Full Text

19. Carette JE, Raaben M, Wong AC, et al.: Ebola virus entry requires the cholesterol transporter Niemann-Pick C1. Nature. 2011; 477(7364): 340-3. PubMed Abstract | Publisher Full Text | Free Full Text

20. Côté M, Misasi J, Ren T, et al.: Small molecule inhibitors reveal Niemann-Pick C1 is essential for Ebola virus infection. Nature. 2011; 477(7364): 344-8. PubMed Abstract | Publisher Full Text | Free Full Text

21. Yonezawa A, Cavrois M, Greene WC: Studies of Ebola virus glycoproteinmediated entry and fusion by using pseudotyped human immunodeficiency virus type 1 virions: involvement of cytoskeletal proteins and enhancement by tumor necrosis factor alpha. $J$ Virol. 2005; 79(2): 918-26. PubMed Abstract | Publisher Full Text | Free Full Text

22. Panchal RG, Reid SP, Tran JP, et al:: Identification of an antioxidant smallmolecule with broad-spectrum antiviral activity. Antiviral Res. 2012; 93(1): 23-9. PubMed Abstract | Publisher Full Text

23. Selaković Z, Opsenica D, Eaton B, et al:: A limited structural modification results in a significantly more efficacious diazachrysene-based filovirus inhibitor. Viruses. 2012; 4(8): 1279-88.

PubMed Abstract | Publisher Full Text | Free Full Text

24. Yermolina MV, Wang J, Caffrey M, et al: Discovery, synthesis, and biological evaluation of a novel group of selective inhibitors of filoviral entry. $J$ Med Chem. 2011; 54(3): 765-81.

PubMed Abstract | Publisher Full Text | Free Full Text

25. Kesel AJ, Huang Z, Murray MG, et al.: Retinazone inhibits certain blood-borne human viruses including Ebola virus Zaire. Antivir Chem Chemother. 2014 23(5): 197-215

PubMed Abstract | Publisher Full Text

26. Basu A, Li B, Mills DM, et al.: Identification of a small-molecule entry inhibitor for filoviruses. J Virol. 2011; 85(7): 3106-19. PubMed Abstract | Publisher Full Text | Free Full Text

27. Bishop BM: Potential and emerging treatment options for Ebola virus disease 
Ann Pharmacother. 2015; 49(2): 196-206.

PubMed Abstract | Publisher Full Text

28. Cunninham J, Lee K, Ren T, et al.: Small molecules inhibitors of Ebola and Lassa fever viruses. 2014.

Reference Source

29. Panchal RG, Reid SP, Tran JP, et al.: Identification of an antioxidant smallmolecule with broad-spectrum antiviral activity. Antiviral Res. 2012; 93(1): 23-9. PubMed Abstract | Publisher Full Text

30. Dube D, Schornberg KL, Shoemaker CJ, et al.: Cell adhesion-dependent membrane trafficking of a binding partner for the ebolavirus glycoprotein is a determinant of viral entry. Proc Natl Acad Sci USA. 2010; 107(38): 16637-42. PubMed Abstract | Publisher Full Text | Free Full Text

31. Yonezawa A, Cavrois M, Greene WC: Studies of Ebola virus glycoproteinmediated entry and fusion by using pseudotyped human immunodeficiency virus type 1 virions: involvement of cytoskeletal proteins and enhancement by tumor necrosis factor alpha. J Virol. 2005; 79(2): 918-26. PubMed Abstract | Publisher Full Text | Free Full Text

32. Fakhfakh MA, Fournet A, Prina E, et al:: Synthesis and biological evaluation of substituted quinolines: potential treatment of protozoal and retroviral coinfections. Bioorg Med Chem. 2003; 11(23): 5013-23. PubMed Abstract | Publisher Full Text

33. Fournet A, Mahieux R, Fakhfakh MA, et al:: Substituted quinolines induce inhibition of proliferation of HTLV-1 infected cells. Bioorg Med Chem Lett. 2003; 13(5): 891-4.

PubMed Abstract | Publisher Full Text

34. Gantier JC, Fournet A, Munos MH, et al.: The effect of some 2-substituted quinolines isolated from Galipea longiflora on Plasmodium vinckei petter infected mice. Planta Med. 1996; 62(3): 285-6. PubMed Abstract | Publisher Full Text

35. Campos Vieira N, Vacus J, Fournet A, et al:: Antileishmanial activity of a formulation of 2-n-propylquinoline by oral route in mice model. Parasite. 2011; 18(4): 333-6.

PubMed Abstract | Publisher Full Text | Free Full Text

36. Nakayama H, Loiseau PM, Bories C, et al:: Efficacy of orally administered 2-substituted quinolines in experimental murine cutaneous and visceral leishmaniases. Antimicrob Agents Chemother. 2005; 49(12): 4950-6. PubMed Abstract | Publisher Full Text | Free Full Text

37. Schmidt $F$, Champy $\mathrm{P}$, Séon-Méniel $\mathrm{B}$, et al.: Chemicals possessing a neurotrophin-like activity on dopaminergic neurons in primary culture. PLOS One. 2009; 4(7): e6215.

PubMed Abstract | Publisher Full Text | Free Full Text

38. Perovic VR: Virtual screen for candidate inhibitors of EBOLA virus infection. F1000Res. 2015.

Reference Source

39. Veljkovic V, Loiseau PM, Figadère B, et al:: Dataset 1 in: Virtual screen for repurposing approved and experimental drugs for candidate inhibitors of EBOLA virus infection. F1000Research. 2015. Data Source

40. Veljkovic V, Loiseau PM, Figadère B, et al.: Dataset 2 in: Virtual screen for repurposing approved and experimental drugs for candidate inhibitors of EBOLA virus infection. F1000Research. 2015. Data Source

41. Veljkovic V, Loiseau PM, Figadère B, et al.: Dataset $\mathbf{3}$ in: Virtual screen for repurposing approved and experimental drugs for candidate inhibitors of EBOLA virus infection. F1000Research. 2015. Data Source

42. Veljkovic V, Loiseau PM, Figadère B, et al.: Dataset 4 in: Virtual screen for repurposing approved and experimental drugs for candidate inhibitors of EBOLA virus infection. F1000Research. 2015. Data Source 


\section{Open Peer Review}

\section{Current Peer Review Status:}

\section{Version 1}

Reviewer Report 11 February 2015

https://doi.org/10.5256/f1000research.6544.r7633

(C) 2015 Botta B. This is an open access peer review report distributed under the terms of the Creative Commons Attribution License, which permits unrestricted use, distribution, and reproduction in any medium, provided the original work is properly cited.

\section{Bruno Botta}

Department of Chemistry and Pharmaceutical Technology, Sapienza University of Rome, Rome, Italy

To identify drug candidates against Ebola virus infections is surely an urgent need, especially in light of recent virus outbreaks registered mostly in Africa. In this respect, Velijkovic's article is presented in a timely manner and offers a fast and reliable opportunity to screen among large databases to reposition old drugs against Ebola.

The experimental design relies on a consolidated methodology, developed by some of the authors and successfully applied in multiple projects. Overall, the manuscript is clear and few minor editing would be necessary, in my personal opinion, to improve its consistency.

In Materials and Methods, a dataset of 152 drugs that counteract Ebola virus in vitro and in vivo has been selected as training set. However, it seems that an inconsistency does exist within this number. As authors have reported, these anti-Ebola drugs have been identified by Madrid (24 molecules) and Kouznetsova (53 molecules and 95 weaker drugs). Accordingly, the total number of FDA-approved drugs identified in these studies is 172 . Why authors used a smaller set of 152 ? Is there any structural redundancy? A clarification of this discrepancy would improve the reproducibility of the work.

Finally, if I understood properly authors have selected more than 500 molecules (including FDAapproved and experimental drugs) as anti-Ebola candidates by means of in silico screening and suggest that further in vitro/in vivo tests should be performed on these molecules. In my opinion, this number is still too large for enabling efficient and fast in vitro and/or in vivo assays.

Experimental testing of this set would require significant efforts. Just for comparison, the number of candidates selected in silico by authors is about half of those selected by Madrid by means of HTS (ref 2). Is there a way to prioritize small molecules by using the EIIP/AQVN-based approach, and to provide a lower number of compounds to be submitted to experimental evaluation? Authors should comment on this point, because the advantage of using the EIIP/AQVN-based screening in silico appears to be limited in the current version of the manuscript. 
Competing Interests: No competing interests were disclosed.

I confirm that I have read this submission and believe that I have an appropriate level of expertise to confirm that it is of an acceptable scientific standard.

Author Response 13 Feb 2015

Veljko Veljkovic, University of Belgrade, Institute of Nuclear Sciences VINCA, P.O. Box 522, 11001 Belgrade, Serbia

The aim of the work was not only to reduce the number of candidate drugs for EVD but to select all approved drugs which will efficiently target GP or its receptor. Further filtering of these candidate anti-Ebola drugs by other structural tools (pharmacophoric modeling, docking studies, etc.) will reduce the number of compounds for experimental testing.

Competing Interests: No competing interests were disclosed.

Reviewer Report 11 February 2015

https://doi.org/10.5256/f1000research.6544.r7552

(C) 2015 Butaye P. This is an open access peer review report distributed under the terms of the Creative Commons Attribution License, which permits unrestricted use, distribution, and reproduction in any medium, provided the original work is properly cited.

\section{Patrick Butaye}

School of Veterinary Medicine, Ross University, Basseterre, Saint Kitts and Nevis

This manuscript deals with the in silico analysis of molecules for their activity against Ebola Virus (EBV). They started from a reference library of compounds who have previously demonstrated in vitro and/or in vivo activity against EBV and analyzed these compounds by the determination of their EIIP and AQVN. With these data, they scanned a larger collection of compounds with unknown activity against EBV and selected possible candidates to test for their activity in vitro and/or in vivo. This is a straight forward manuscript however it may be better structured. The part of the M\&M dealing with the EIIP and AQVN is more appropriate to go into the introduction since this is background information of the calculation. For clarity of the manuscript is also better to separate the results section as it is difficult to follow now. Start with the analysis of the compounds with known activity, the two datasets, and then proceed with results from the unknown dataset. Then in the discussion, the different products of interest may be evaluated. This will largely increase the readability. Upon the antibiotics, it would be good to elaborate a bit on how they work on EBV, since common sense tells that antibiotics do not work on viruses. A better explanation on how these products may interact and inhibit/kill EBV would also be good.

Competing Interests: No competing interests were disclosed.

I confirm that I have read this submission and believe that I have an appropriate level of 
expertise to confirm that it is of an acceptable scientific standard.

Author Response 13 Feb 2015

Veljko Veljkovic, University of Belgrade, Institute of Nuclear Sciences VINCA, P.O. Box 522, 11001 Belgrade, Serbia

The antibiotics presented in this article are not selected because of their antibiotic activity but they are proposed as candidate entry inhibitors of Ebola virus (drug repurposing).

Competing Interests: No competing interests were disclosed.

The benefits of publishing with F1000Research:

- Your article is published within days, with no editorial bias

- You can publish traditional articles, null/negative results, case reports, data notes and more

- The peer review process is transparent and collaborative

- Your article is indexed in PubMed after passing peer review

- Dedicated customer support at every stage

For pre-submission enquiries, contact research@f1000.com 